Bundesgesundheitsbl 2020 63:987-996 https://doi.org/10.1007/s00103-020-03178-9 Online publiziert: 2 . Juli 2020

(c) Der/die Autor(en) 2020
Jördis Wothge ${ }^{1} \cdot$ Hildegard Niemann ${ }^{2}$

'Fachgebiet I 2.4, Lärmminderung bei Anlagen und Produkten, Lärmwirkungen, Umweltbundesamt, Dessau-Roßlau, Deutschland

${ }^{2}$ Fachgebiet 24, Gesundheitsberichterstattung, Robert Koch-Institut, Berlin, Deutschland

\title{
Gesundheitliche Auswirkungen von Umgebungslärm im urbanen Raum
}

\section{Einleitung}

Umgebungslärm wird in Deutschland im Rahmen des Bundes-Immissionsschutzgesetzes in $\$ 47 \mathrm{~b}$ beschrieben als „belästigende oder gesundheitsschädliche Geräusche im Freien, die durch Aktivitäten von Menschen verursacht werden, einschließlich des Lärms, der von Verkehrsmitteln, Straßenverkehr, Eisenbahnverkehr, Flugverkehr sowie Geländen für industrielle Tätigkeiten ausgeht" [1]. In Städten ist die Belastung der Menschen durch Umgebungslärm und insbesondere Verkehrslärm ${ }^{1}$ hoch. So ist in Deutschland etwa jede fünfte Person durch Verkehrslärm belastet [2]. Neben Lärmbelästigung, Schlafstörungen und Beeinträchtigungen in der kognitiven Entwicklung kann eine andauernde langjährige Geräuschbelastung unter anderem HerzKreislauf-Erkrankungen zur Folge haben und Depressionen begünstigen. Insbesondere im urbanen Raum ist Lärm durch unterschiedliche Schallquellenarten ein ständiger Begleiter. Der vorliegende Artikel beschreibt die Verkehrslärmbelastung in Deutschland beruhend auf den Daten aus der Umgebungslärmrichtlinie, gibt einen Überblick über die wichtigsten gesundheitlichen Folgen einer hohen Geräuschbelastung, thematisiert die Her-

\footnotetext{
1 Lärm ist unerwünschter Hörschall bzw. Hörschall der zu Gesundheitsgefahren führen kann. In diesem Artikel wird der Begriff Lärm auch im Zusammenhang mit Verkehrsgeräuschen genutzt, da Verkehrsgeräusche von den Betroffenen ganz überwiegend als unerwünschte Geräusche erlebt werden.
}

ausforderung von Umgebungslärm im städtischen Raum und zeigt mögliche Maßnahmen zur Lärmminderung in der Stadt auf.

\section{Verkehrslärmbelastung in Deutschland (mit Fokus auf den urbanen Raum)}

Beruhend auf den Berechnungsverfahren der Umgebungslärmrichtlinie (2002/49/EG; [3]) wird alle 5 Jahre die Geräuschbelastung durch unterschiedliche Schallquellen berechnet und kartiert. Im Einzelnen werden in Deutschland 70 Ballungsräume mit rund 24,4 Mio. Einwohnerinnen und Einwohnern kartiert sowie $49.000 \mathrm{~km}$ Hauptverkehrsstraßen, $14.000 \mathrm{~km}$ Haupteisenbahnstrecken und alle 11 Großflughäfen. Die Daten der letzten Erhebungsrunde aus dem Jahr 2017 zeigen, dass in Deutschland ca. 10,9 Mio. Menschen durch Verkehrslärm von durchschnittlich mehr als $50 \mathrm{~dB}$ $L_{\text {night }}{ }^{2}$ in der Nacht betroffen sind. Etwa $15,8 \mathrm{Mio}$. sind bei einer ganztägigen Betrachtung von $24 \mathrm{~h}$ durchschnittlich durch Verkehrslärm von mehr als $55 \mathrm{~dB}$

\footnotetext{
2 Der $L_{\text {night }}$ ist ein A-bewerteter Mittelungspegel für die Nachtzeit (Night Level; in Deutschland: 22-6 Uhr) in dB.

${ }^{3}$ Der $L_{\text {den }}$ ist ein A-bewerteter Tag-AbendNacht-Schallpegel (Day-Evening-Night Level), Mittelungspegel für $24 \mathrm{~h}$ mit einem Zuschlag von $5 \mathrm{~dB}(\mathrm{~A})$ für die Abendzeit (in Deutschland: 18-22 Uhr) und von $10 \mathrm{~dB}$ für die Nachtzeit (in Deutschland:22-6Uhr) in dB.
}

$L_{\mathrm{den}}{ }^{3}$ belastet. ${ }^{4}$ Dies bedeutet, dass 13,2 \% der Bevölkerung in Deutschland durch nächtlichen und 19,1\% der Bevölkerung durch ganztägigen Verkehrslärm oberhalb dieser Dauerschallpegel betroffen sind (• Abb. 1; [2]). Bei gesonderter Betrachtung urbaner Räume, die in der Umgebungslärmrichtlinie Ballungsräume genannt werden, zeigt sich, dass von den 24,4 Mio. Menschen, die in Deutschland in Ballungsräumen leben, 9,3 Mio. Menschen von mehr als $55 \mathrm{~dB} L_{\mathrm{den}}$ über den ganzen Tag verteilt belastet sind und 5,7 Mio. Menschen durch nächtlichen Verkehrslärm von mehr als $50 \mathrm{~dB} L_{\text {night. }} \$ 47 \mathrm{~b}$ des BundesImmissionsschutzgesetzes definiert Ballungsräume in Deutschland als Gebiete, in denen die Zahl der Einwohnerinnen und Einwohner über 100.000 Menschen beträgt und die Bevölkerungsdichte über 1000 Einwohnerinnen und Einwohner pro Quadratkilometer liegt [1].

Dabei gehen von den jeweiligen Verkehrsträgern unterschiedliche Belastungen aus: Die am weitesten verbreitete Verkehrslärmquelle ist der Straßenverkehr. Dies gilt insbesondere für den urbanen Raum, hier ist die Zahl der Straßenverkehrslärmbelasteten mit etwa 5,7 Mio. belasteten Personen über $55 \mathrm{~dB}$ tagsüber und 1,7 Mio. belasteten Personen über $50 \mathrm{~dB}$ nachts in etwa zweimal so hoch wie außerhalb von Ballungsräumen (• Tab. 1). Der Schienenverkehr ist im

\footnotetext{
${ }^{4}$ Die Werte $50 \mathrm{~dB} L_{\text {den }}$ und $55 \mathrm{~dB} L_{\text {night }}$ entsprechen den niedrigsten Werten, die nach den Vorgaben der Umgebungslärmrichtlinie zu kartieren sind [3].
} 


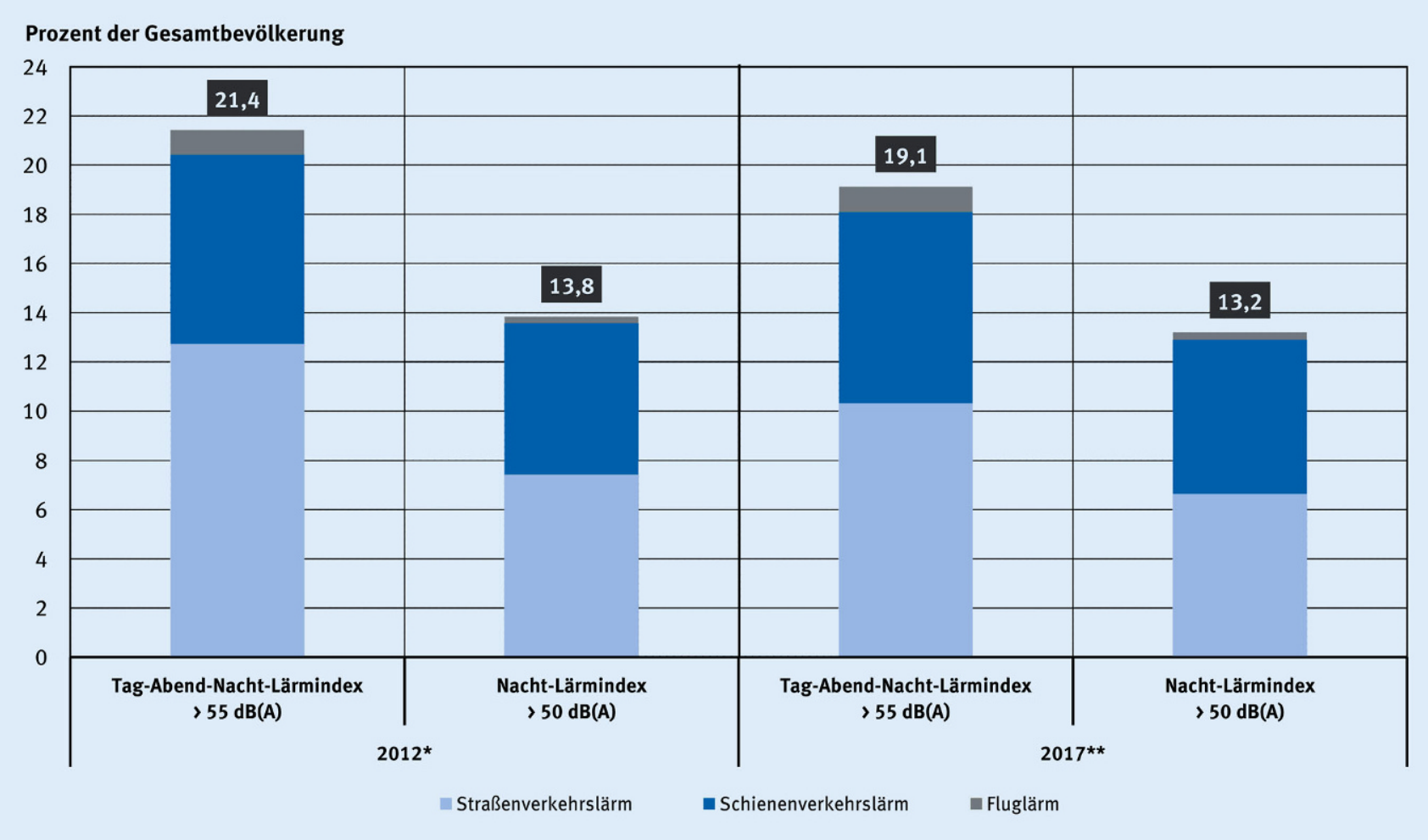

Abb. $1 \Delta$ Anteil der durch Verkehrslärm belasteten Bevölkerung in Deutschland. Vergleich der Jahre 2012 und 2017. Asterisk Auswertungsstand der Lärmkartierung 2012: 29.02.2016, zwei Asteriske Auswertungsstand der Lärmkartierung 2017: 30.12.2019. (Quelle: [2], Daten der Lärmkartierungen 2012 und 2017, berechnet aus Mitteilungen der Bundesländer und des Eisenbahn-Bundesamtes entsprechend § $47 \mathrm{c}$ BImSchG, eigene Zusammenstellung)

Bereich der Schienenwege insbesondere nachts ein Problem. Von Fluglärm sind bundesweit betrachtet weniger Menschen betroffen, im Umfeld von Verkehrsflughäfen ist der Fluglärm jedoch häufig die dominante Geräuschquelle.

Da im Rahmen der „Lärmkartierung“ ausschließlich Hauptverkehrsstraßen mit einem Verkehrsaufkommen von mehr als 3 Mio. Kraftfahrzeugen pro Jahr, Haupteisenbahnstrecken mit mehr als 30.000 Zugbewegungen jährlich sowie Großflughäfen mit mehr als 50.000 Flugbewegungen im Jahr berücksichtigt werden, stellt die Gesamtzahl der Betroffenen eine Unterschätzung dar.

\section{Gesundheitliche Auswirkungen von Umgebungslärm}

Eine dauerhafte Belastung durch Umgebungslärm kann weitreichende gesundheitliche Folgen haben. Die Weltgesundheitsorganisation (WHO) definiert Gesundheit „nicht lediglich als die Abwesenheit von Krankheit, son- dern als einen Zustand vollkommenen physischen, geistigen und sozialen Wohlbefindens" [4]. Beruhend auf dieser Definition identifizieren die neuen WHO-Leitlinien für Umgebungslärm für die europäische Region verschiedene gesundheitliche Auswirkungen. Dazu zählen unter anderem Lärmbelästigung, Schlafstörungen, kognitive Beeinträchtigungen, Herz-Kreislauf-Erkrankungen und mentale Auswirkungen [5]. Insbesondere die Lärmbelästigung und Schlafstörungen können bei längerer Latenz $\mathrm{zu}$ weiteren manifesten gesundheitlichen Erkrankungen führen.

Die gesundheitlichen Folgen von Umgebungslärm finden auch Eingang in die europäische Gesetzgebung. Anhang III der Umgebungslärmrichtlinie (2002/49/EG) sieht die Bewertung der Auswirkungen von Umgebungslärm auf die Bevölkerung mithilfe von Expositions-Wirkungs-Funktionen vor [3]. Die Expositions-Wirkungs-Funktionen stellen den Zusammenhang zwischen der Intensität der Dauerschallbelastung (z. B.
$L_{\text {den }}$ oder $\left.L_{\text {night }}\right)$ und einer gesundheitlichen Auswirkung (z. B. Lärmbelästigung, selbstberichteten Schlafstörungen oder ischämischen Herzerkrankungen) dar. Die Umgebungslärmrichtlinie gibt keine Grenzwerte vor, die für die Mehrheit der Bevölkerung einen vorsorgenden $\mathrm{Ge}$ sundheitsschutz erzielen könnten. Der Anhang III dient der Information über die gesundheitlichen Auswirkungen, die mit der im Rahmen der Richtlinie berechneten Geräuschbelastung zu assoziieren ist.

\section{Lärmbelästigung}

Belästigung ist neben Schlafstörungen die häufigste unmittelbare Auswirkung von Umgebungslärm. So fühlen sich beispielsweise etwa $75 \%$ der Bevölkerung in Deutschland durch Straßenverkehrslärm belästigt (• Abb. 2; [6]). Laut WHO ist die Belästigung eine komplexe Reaktion, die primär aus drei Elementen besteht: (a) einer häufig wiederholten Störungvon Aktivitäten (wie beispielsweise der Kom- 
munikation mit anderen Menschen, dem Hören von Musik, dem Lesen, Arbeiten oder Schlafen), die Verhaltensanpassungen zwecks Minimierung der Störung nach sich zieht; (b) eine einstellungsbezogene Reaktion (Wut oder Ärger über die Störung oder die negative Evaluation der entsprechenden Geräuschquelle) und (c) eine kognitive Reaktion (beispielsweise die Einsicht, dass es kaum eine Möglichkeit gibt, sich gegen die ungewollte Situation zu wehren). Es handelt sich hierbei um eine Stressreaktion die aufgrund eines Umweltstressors (unerwünschte Geräusche bzw. Lärm) ausgelöst wird und physiologische, emotionale, kognitive und Verhaltensänderungen nach sich zieht. Sie kann anteilig erinnert und in Form der langfristigen Belästigung verbalisiert werden $[7,8]$.

Die Lärmbelästigung kann ein Vermittler für die Manifestation weiterer (insbesondere klinischer) gesundheitlicher Auswirkungen sein. Die Lärmbelästigung wird durch ein standardisiertes Fragebogeninstrument erhoben - die sogenannte ICBEN-Frage ${ }^{5}$. Diese differenziert das Stör- und Belästigungserleben der letzten 12 Monate durch eine Geräuschquelle anhand von zwei unterschiedlichen Skalen - einer 11-stufigen numerischen und einer 5-stufigen verbalen Skala. Die 5-stufige verbale Skala, reicht von „überhaupt nicht“ (1) bis „äußerst“ (5; [9]). Das Belästigungsurteil beschreibt somit nicht die momentane Belästigung, sondern ein längerfristiges Lärmerleben im individuellen Kontext. Für die Darstellung des ExpositionsWirkungs-Zusammenhangs wird der prozentuale Anteil an hoch belästigten Personen pro Schallpegel berechnet. Als „hoch belästigt" werden hierbei in vielen Studien die obersten drei Antwortkategorien der 11-stufigen numerischen Skala

\footnotetext{
5 ICBEN steht für International Commission on the Biological Effects of Noise. Die Kommission traf 1999 eine Vereinbarung über die Frage zur Erfassung der generellen und quellenspezifischen Lärmbelästigung; diese international vereinheitlichte Frage wird heute oftmals vereinfacht als "die ICBEN-Frage“ bezeichnet. Die Fragestellung ist in mehrere Sprachen übersetzt und international standardisiert worden in der Norm ISO/TS 15666 [9].
}

Bundesgesundheitsbl 2020 -63:987-996 https://doi.org/10.1007/s00103-020-03178-9

(c) Der/die Autor(en) 2020

\section{J. Wothge $\cdot$ H. Niemann}

\section{Gesundheitliche Auswirkungen von Umgebungslärm im urbanen Raum}

\section{Zusammenfassung}

Umgebungslärm ist eine große Belastung für die Menschen in Deutschland, insbesondere im urbanen Raum. Häufig sind mehrere Schallquellenarten für die Belastung verantwortlich. Die andauernde Belastung kann verschiedene und weitreichende gesundheitliche Auswirkungen haben wie Lärmbelästigung, Schlafstörungen, ischämische Herzerkrankungen und Depressionen. Zusätzlichen Belastungen entstehen z. B. durch Feinstaub oder Luftschadstoffe. Um den Herausforderungen im urbanen Raum zu begegnen, lassen sich verschiedene Maßnahmen und Instrumente beschreiben und diskutieren. Der vorliegende Beitrag gibt einen Überblick über wichtige gesundheitliche Folgen einer langfristigen Geräuschbelastung, thematisiert die verbundenen Herausforderungen im städtischen Raum und zeigt Möglichkeiten zur Lärmminderung auf.

Schlüsselwörter

Verkehrslärm · Umgebungslärmrichtlinie . Lärmwirkungen · Umwelt und Gesundheit . Urbanes Leben

\section{Adverse health effects due to environmental noise exposure in urban areas}

\section{Abstract}

Environmental noise is a great burden for the population in Germany, especially in urban areas. People are often exposed not only to one but several noise sources. Longterm exposure to environmental noise can have several and severe adverse effects on human health, such as noise annoyance, sleep disturbances, ischaemic heart disease and depression. Additional burdens arise from multiple exposure to particulate matter or air pollutants. The current article describes the relevant adverse health effects due to long-term environmental noise exposure, discusses the challenges of environmental noise in urban areas different and introduces measures and instruments to abate environmental noise.

\section{Keywords}

Traffic noise - Environmental noise directive . Noise impact - Environment and health . Urban life definiert. Dies entspricht den obersten $27 \%$ der Skala [10].

Die Belästigungsangaben durch einzelne Schallquellen unterscheiden sich zum Teil deutlich. So gilt Fluglärm als die am stärksten belästigende Verkehrslärmquelle, gefolgt von Straßen und Schienenverkehrslärm [10]. Studien der letzten Jahre weisen inzwischen darauf hin, dass das Belästigungserleben bei hohen Schallpegeln durch Schienenverkehr als stärker belästigend im Vergleich zu Straßenverkehrslärm wahrgenommen wird $[11,12]$. Das unterschiedliche Belästigungspotenzial spiegelt sich auch in den Expositions-Wirkungs-Funktionen der neuen WHO-Leitlinien für Umgebungslärm für die europäische Region deutlich wider. So sind $10 \%$ der Personen bei einem durchschnittlichen ganztägigen Dauerschallpegel von $45 \mathrm{~dB} L_{\mathrm{den}}$ des Luftverkehrs hoch belästigt. Beim Straßenverkehr ist dieser Prozentsatz bei einer durchschnittlichen Dauerschallbelastung von $53 \mathrm{~dB} L_{\mathrm{den}}$ und beim Schienenverkehr bei einer durchschnittlichen Dauerschallbelastung von $54 \mathrm{~dB} L_{\mathrm{den}}$ erreicht [5]. Neben dem hier beschriebenen Verkehrslärm stellt, insbesondere im urbanen Raum, der Nachbarschaftslärm eine weitere wichtige Quelle der Belästigung dar.

\section{Schlafstörungen}

Schlaf ist für den Menschen unverzichtbar. Er dient der psychischen und physischen Erholung und der „Ent- 
Tab. 1 Die Anzahl verkehrslärmbelasteter Personen innerhalb und außerhalb von Ballungsräumen in Deutschland

\begin{tabular}{|c|c|c|c|c|}
\hline & \multicolumn{2}{|c|}{ Innerhalb von Ballungsräumen } & \multicolumn{2}{|c|}{ Außerhalb von Ballungsräumen } \\
\hline & $\begin{array}{l}L_{\mathrm{den}} \\
>55 \mathrm{~dB}\end{array}$ & $\begin{array}{l}L_{\text {night }} \\
>50 \mathrm{~dB}\end{array}$ & $\begin{array}{l}L_{\mathrm{den}} \\
>55 \mathrm{~dB}\end{array}$ & $\begin{array}{l}L_{\text {night }} \\
>50 d B\end{array}$ \\
\hline Straßenverkehr & 5.717 .500 & 3.777 .600 & 2.788 .700 & 1.706 .200 \\
\hline Schienenverkehr & 3.131 .800 & 2.428 .500 & 3.304 .100 & 2.735 .400 \\
\hline Luftverkehr & 530.500 & 147.600 & 285.000 & 92.900 \\
\hline Gesamt & 9.379 .800 & 6.353 .700 & 6.377 .800 & 4.534 .500 \\
\hline \multicolumn{5}{|c|}{$\begin{array}{l}d B \text { Dezibel, } L_{\text {night }} \text { A-bewerteter Mittelungspegel für die Nachtzeit (Night Level; in Deutschland: } \\
22-6 \text { Uhr) in dB, } L_{\text {den }} \text { A-bewerteter Tag-Abend-Nacht-Schallpegel (Day-Evening-Night Level), } \\
\text { Mittelungspegel für } 24 \mathrm{~h} \text { mit einem Zuschlag von } 5 \mathrm{~dB}(\mathrm{~A}) \text { für die Abendzeit (in Deutschland: } \\
18-22 \text { Uhr) und von } 10 \mathrm{~dB} \text { für die Nachtzeit (in Deutschland: } 22-6 \text { Uhr) in } \mathrm{dB} ; 50 \mathrm{~dB} L_{\text {den }} \text { und } 55 \mathrm{~dB} \\
L_{\text {night }}=\text { niedrigste Werte, die nach den Vorgaben der Umgebungslärmrichtlinie zu kartieren sind [3] }\end{array}$} \\
\hline
\end{tabular}

müdung“. Ein durch Umgebungslärm gestörter oder zu kurzer Schlaf kann das neuroendokrine System einschließlich des Immunsystems negativ beeinflussen. Langfristig ist der durch Geräusche gestörte Schlaf daher, neben der Beeinträchtigung geistiger und körperlicher Leistungen als akute Wirkung, auch mit einer erhöhten kardiometabolischen Morbidität und Mortalität verbunden (z.B. [13-15]). Eine neue Studie weist darauf hin, dass insbesondere eine gestörte Nachtruhe das Risiko erhöht, eine Herz-Kreislauf-Erkrankung zu entwickeln. Wesentliche Einflussfaktoren in diesem Prozess sind die Bildung von freien Radikalen (oxidativer Stress) und Entzündungsreaktionen in Gehirn, Herz und Gefäßen [16]. Ebenfalls medizinisch relevant sind psychische Erkrankungen wie Depression und Angststörungen, die häufig durch gestörten Schlaf ausgelöst werden. Die durch Stress (mit-)verursachten Erkrankungen zeigen sich oft erst nach einer Latenzzeit von 10-15 Jahren.

Der „Goldstandard“ für die Messung des Schlafs ist die Polysomnographie. Mit ihrer Hilfe kann für die schlafende Person ein individuelles Schlafprofil erstellt werden. Die Auswertung solcher Schlafprofile zeigt, dass sich wiederholende oder andauernde nächtliche Schallreize als fragmentierter Schlafver-

6 In der Schlafmedizin bezeichnet „Arousal“ eine zeitlich eng begrenzte Zustandsänderung, die den Organismus von einem niedrigen Erregungsniveau auf ein höheres anhebt. Die American-Sleep-Disorder-Association fordert fürEEG-Arousal eine Episode von 3-30 s. lauf einschließlich von Arousal ${ }^{6}$ und Aufwachreaktionen ${ }^{7}$ äußern.

In den letzten Jahren wurde, insbesondere durch Feldstudien, verstärkt der Zusammenhang zwischen einzelnen Schallereignissen (Maximalpegel, $L_{\max }$ ) und einem sich anschließenden Erwachen im Schlafprofil in den Blick genommen (z. B. [18, 19]). Aus den Studien wurden mittels Maximalschallpegeln Expositions-Wirkungs-Beziehungen für Einzelschallereignisse des Verkehrslärms abgeleitet (z. B. [20, 21]; • Abb. 3). Um andere Einflüsse als den nächtlichen Lärm so gering wie möglich zu halten, wurden ausschließlich (schlaf-)gesunde Personen als freiwillige Probandinnen und Probanden in den Studien untersucht. Aufgrund dieser starken Selektion und der sehr kleinen Stichprobengrößen ist daher eine Übertragung der Ergebnisse auf die Gesamtbevölkerung aus epidemiologischer Sicht nicht möglich [22, 23].

Eine weitere Methode ist die Erhebung der Selbsteinschätzungen von Schlafstörungen (sogenannte selbstberichtete Schlafstörungen). Sie kann in großen Zufallsstichproben erfragt werden (z.B. mittels Fragebögen oder Interview) und ist daher grundsätzlich

\footnotetext{
7 Nach Rechtschaffen und Kehls (1968) liegt eine Aufwachreaktion vor, wenn in einer Auswerteepoche von $30 \mathrm{~s}$ überwiegend EEGWachmuster vorliegen [17]. In Untersuchungen des Deutschen Zentrums für Luft- und Raumfahrt(DLR) werden auch lärmbedingte Änderungen des Schlafstadiums in das Stadium S1 (Einschlafstadium) zu den Aufwachreaktionen gerechnet $[18,19]$.
}

dafür geeignet, generalisierte Expositions-Wirkungs-Beziehungen zu erstellen [5, 24-26]. Da der Mensch während der meisten Zeit des Schlafes ohne Wachbewusstsein ist, lassen sich Fehleinschätzungen (,information bias“) nicht vermeiden. Diese Fehleinschätzungen sind nichtdifferenzielle Fehlklassifikationen und damit nahezu unabhängig von der Geräuschbelastung, sodass dadurch der statistische Zusammenhang der Expositions-Wirkungs-Beziehung grundsätzlich unterschätzt wird [27].

Bei einem Vergleich der Expositions-Wirkungs-Funktionen der WHOMetaanalyse (Studien aus den Jahren 2000-2015; [5]) mit der ExpositionsWirkungs-Funktion von Miedema und Vos aus dem Jahr 2007 (Studien bis zum Jahr 2004; siehe rote Linie in D Abb. 4; [26]) zeigt sich, dass bei gleichem nächtlichen Dauerschallpegel $L_{\text {night }}$ der Anteil sich hochgradig durch Flug- und Schienenverkehrslärm im Schlaf gestört fühlender Menschen (\% HSD [,highly sleep disturbed"]) in den zurückliegenden Jahren erheblich zugenommen hat. In der WHO-Metaanalyse sind keine Ergebnisse von Studien enthalten, die nach 2015 publiziert wurden. Würden diese einbezogen, ist ein weiterer Anstieg der hochgradig schlafgestörten Personen durch Flug- und Schienenverkehrslärm erkennbar [11, 15]. Beim Straßenverkehrslärm ist kein vergleichbarer Anstieg ersichtlich.

Neben der Verkehrslärmbelastung spielt in dicht bebauten urbanen Räumen auch der Nachbarschaftslärm eine wichtige Rolle. So wurde in der WHOLARES-Studie in 8 europäischen Städten der Nachbarschaftslärm etwa gleich häufig als Quelle von Schlafstörungen genannt wie der Verkehrslärm [29]. Geräusche sind dabei nur eine Komponente einer Vielzahl möglicher umweltbedingter Ursachen für Schlafstörungen. Auch Temperatur, Luftfeuchtigkeit oder Lichtverhältnisse sowie eingeschränkte Luftwechsel durch geschlossene Fenster können sich negativ auf den Schlaf des Menschen auswirken ebenso wie innere Ursachen, z. B. Angst, Gesundheitszustand oder schlechte Träume. 


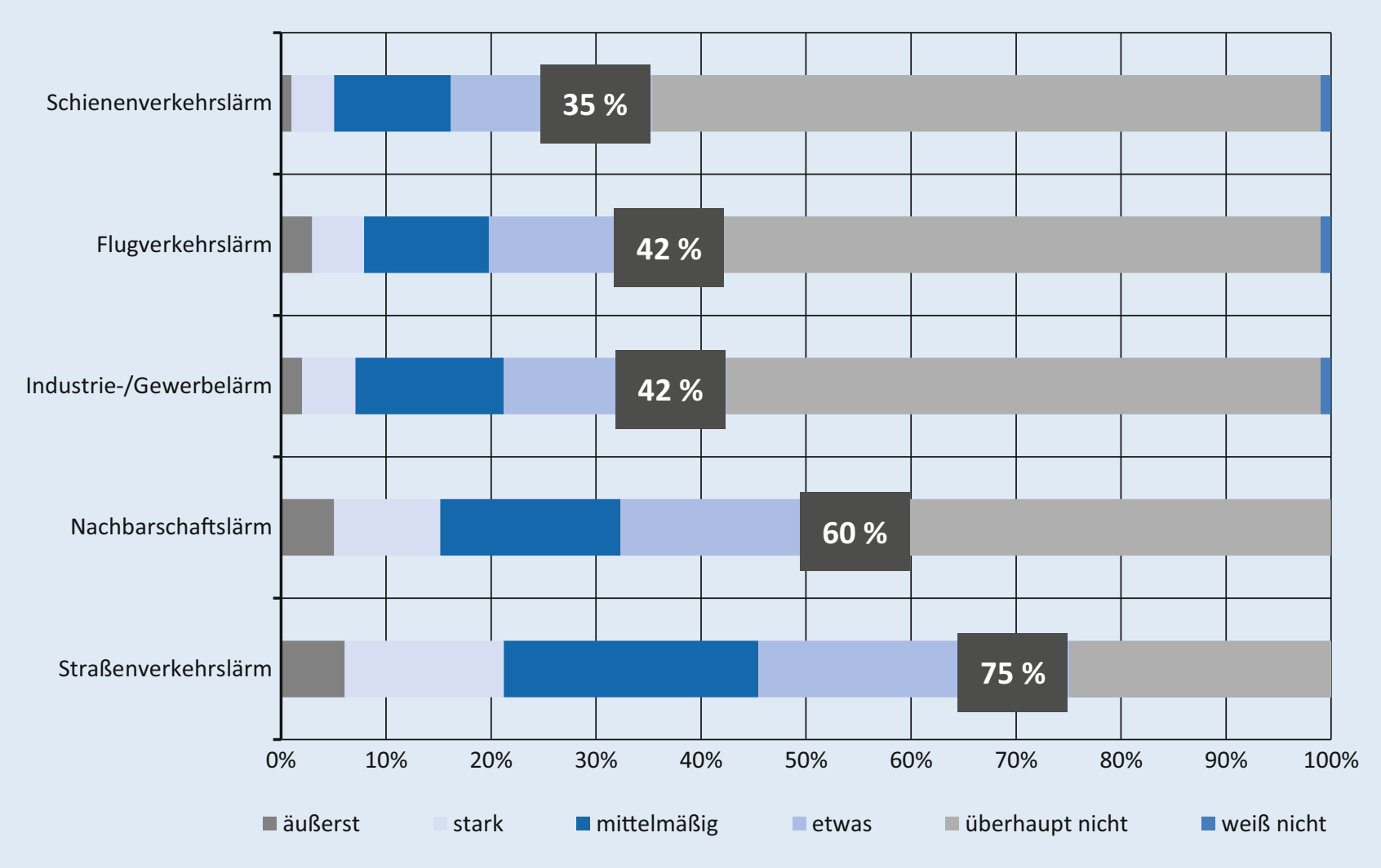

Abb. $2 \triangle$ Ausmaß der Lärmbelästigung der Bevölkerung in Deutschland durch unterschiedliche Lärmquellen. Lärmbelästigung in Deutschland (in \%). Frage: Wenn Sie einmal an die letzten 12 Monate hier bei Ihnen denken, wie stark haben Sie sich persönlich durch den Lärm von folgenden Dingen gestört oder belästigt gefühlt? (Angaben in Prozenten, Abweichungen von 100 Prozenten rundungsbedingt). (Quelle: [6])

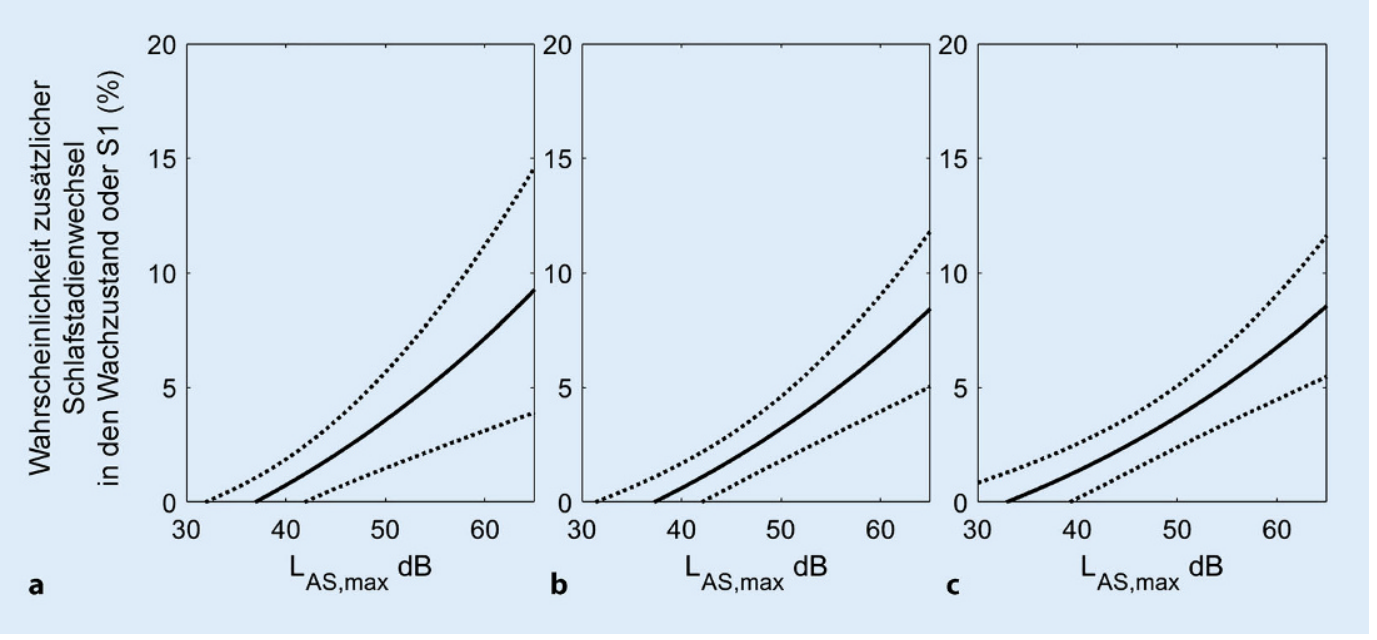

Abb. 3 ॥ Wahrscheinlichkeit zusätzlicher Schlafstadienwechsel in den Wachzustand oder das Einschlafstadium (S1) in einem Zeitfenster von 90 s nach Eintritt des Lärmereignisses in Abhängigkeit vom maximalen Schalldruckpegel ( $L_{A S \text {,max }}$ innen) für a Straße (STRAIN und DEUFRAKO, $N=94$ Probanden), b Flugzeug (STRAIN, $N=61$ ) und c Schienenlärm (DEUFRAKO, $N=33$; Müller et al. [19]). Die spontane Aufwachwahrscheinlichkeit wurde abgezogen. Gestrichelte Linien: $95 \%$ Konfidenzintervalle. (Abbildung entnommen aus: Basner und McGuire [20], veröffentlicht unter der CC-BY 4.0 Lizenz (https://creativecommons. org/licenses/by/4.0/). Es wurden keine Änderungen vorgenommen) 

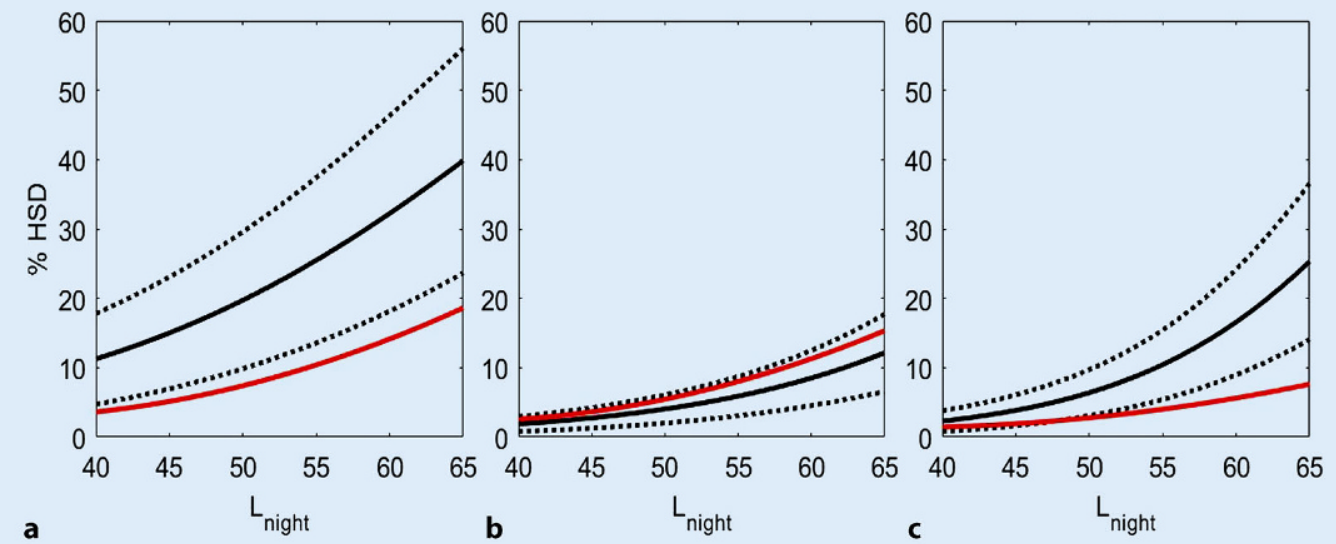

Abb. $4 \Delta$ Anteil hochgradig schlafgestörter Personen (HSD) bei unterschiedlichen Verkehrslärmquellen, basiert auf Antworten zum Erwachen sowie zu Einschlafschwierigkeiten und Schlafstörungen durch Straßenlärm $(N=30.290)$, Schienenlärm $(N=13.640)$ und Fluglärm $(N=12.731)$. Schwarz gestrichelte Linien: $95 \%$ Konfidenzintervalle. Rote Linien: Expositions-Wirkungs-Funktionen Miedema und Vos (2007) zum Vergleich [20, 28]. (Abbildung entnommen aus: Basner und McGuire [20], veröffentlicht unter der CC-BY 4.0 Lizenz (https://creativecommons.org/licenses/by/4.0/). Es wurden keine Änderungen vorgenommen)

\section{Kognitive Beeinträchtigungen (Einschränkung der Entwicklung)}

Bei Kindern laufen grundlegende kognitive Funktionen wie Aufmerksamkeit, Gedächtnis und Sprachverarbeitung weniger automatisiert $\mathrm{ab}$ als bei Erwachsenen und die Möglichkeit, kognitive Bewältigungsstrategien anzuwenden, ist bei ihnen noch nicht voll ausgeprägt. Dies macht sie zu einer besonders vulnerablen Gruppe hinsichtlich kognitiver Beeinträchtigungen. So kann sich Verkehrslärm negativ auf die kognitive Leistungsfähigkeit bei Kindern auswirken, einschließlich einer verschlechterten Langzeitgedächtnisleistung [30, 31]. In den letzten zwei Jahrzehnten sind darüber hinaus vermehrt Untersuchungen zum Zusammenhang zwischen Fluglärm und dem Lese- und Hörverständnis durchgeführt worden. Clark und Paunovic haben in einer Metastudie 2018 beispielsweise insgesamt 14 Studien zum Zusammenhang zwischen Fluglärm und dem Lese- und Hörverständnis analysiert (für Straßenund Schienenverkehrslärm stand keine vergleichbare Evidenz zur Verfügung). 10 dieser Studien zeigten einen signifikanten Zusammenhang zwischen der durchschnittlichen Fluglärmbelastung und einem schlechteren Lese- und Hörverständnis bei Schulkindern [32]. Die
WHO geht davon aus, dass es ab einer durchschnittlichen Geräuschbelastung von $55 \mathrm{~dB} L_{\mathrm{den}} \mathrm{zu}$ einer Verzögerung von einem Monat im Lese- und Hörverständnis kommt [5]. Absolut betrachtet mag diese Zahl zunächst gering erschienen. Ihre Relevanz zeigt sich erst im Vergleich zur Gesamtlernzeit des Erwerbs der Lese- und Hörverständnisses bei Kindern: In der Regel erreichen Schulkinder das Leseverständnis im ersten Schuljahr. Durch Schuleingewöhnung und Ferien liegt die gesamte Lernzeit des ersten Schuljahres bei etwa einem halben Jahr. Eine Verzögerung des Kompetenzerwerbs von einem Monat bedeutet daher eine Verzögerung um etwa ein Sechstel der Leselernzeit gegenüber Kindern, die nicht durch Fluglärm belastet sind. Zum jetzigen Zeitpunkt ist ungeklärt, inwiefern Kinder dieses Defizit kompensieren und wie lange sie dafür benötigen.

\section{Herz-Kreislauf-Erkrankungen}

$\mathrm{Zu}$ den klinisch relevanten gesundheitlichen Auswirkungen des Umgebungslärms zählen insbesondere die HerzKreislauf-Erkrankungen: Umgebungslärm aktiviert das Hormonsystem und das autonome Nervensystem. Infolgedessen können sich der Blutdruck und die Herzfrequenz verändern und der Stoffwechsel und dessen Regulation beeinträchtigt werden. Biologische Risikofaktoren, wie der Blutzucker oder die Gerinnung des Blutes, können beeinflusst werden. Dies kann zu Arterienverkalkung und Bluthochdruck bis hin zum Herzinfarkt führen. - Abb. 5 zeigt ein mögliches Schema, wie eine dauerhafte Geräuschbelastung das Herz-Kreislauf-System sowohl über den direkten als auch den indirekten Pfad beeinträchtigen und welche Auswirkungen dies haben kann. $\mathrm{Zu}$ den relevantesten Herz-Kreislauf-Beeinträchtigungen zählen ischämische Herzkrankheiten, wie Herzinsuffizienz und der Myokardinfarkt, chronische Veränderungen des Blutdrucks, aber auch sogenannte Hirninfarkte bzw. Schlaganfälle. Die Mehrzahl der bisher durchgeführten Studien befasst sich mit dem Zusammenhang von Straßenverkehrs- bzw. Fluglärm auf das Herz-Kreislauf-System [33]. Über den Zusammenhang mit dem Schienenverkehr gibt es hingegen nur wenige Erhebungen und belastbare Erkenntnisse [33]. Die Metaanalyse von van Kempen et al., welche den neuen WHO-Leitlinien für Umgebungslärm für die europäische Region als Grundlage dient, findet beispielsweise einen signifikanten Anstieg des relativen Risikos, geräuschbedingt an einer ischämischen Herzkrankheit zu erkranken von $8 \%$ (Konfidenzintervall $[\mathrm{KI}]$ : $1-15 \%)$ pro $10 \mathrm{~dB} L_{\mathrm{den}}$ des 


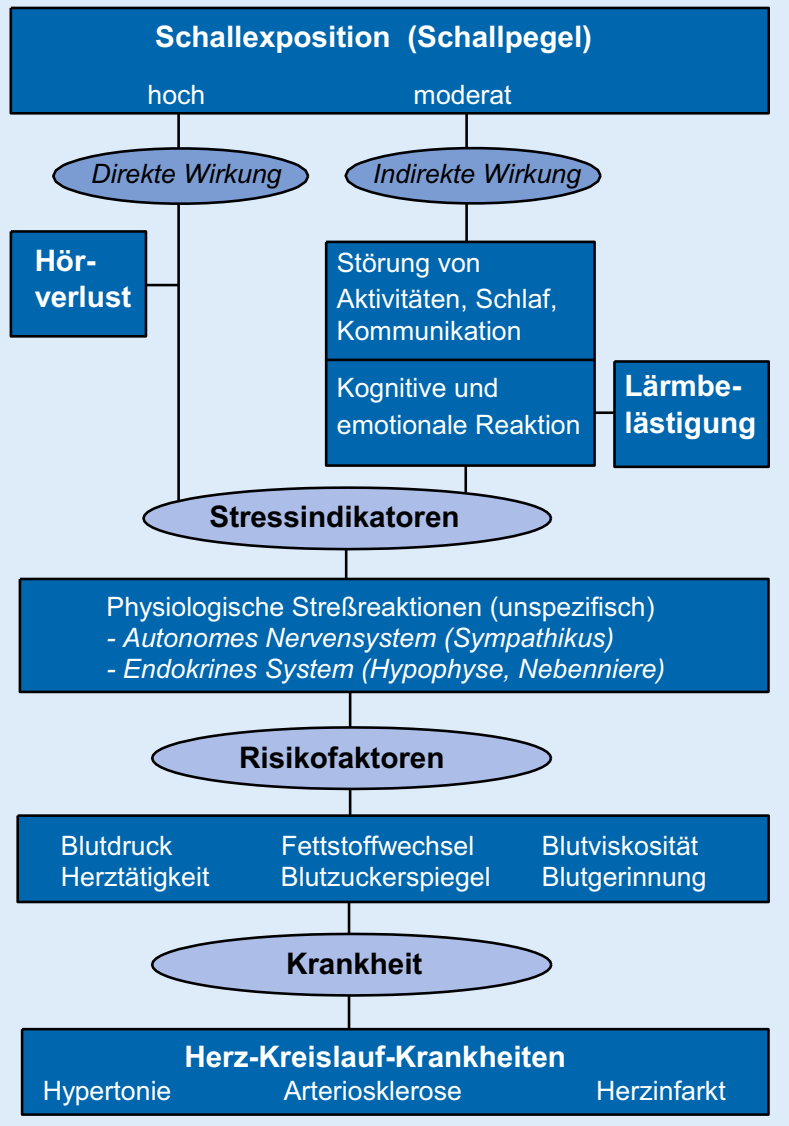

kehrslärm. Ergebnisse der Studie „Gesundheit in Deutschland aktuell 2012“" des Robert Koch-Instituts zeigen zudem, dass eine "starke“ oder „üußerst starke" Lärmbelästigung im Vergleich zu einer Referenzgruppe ohne Lärmbelästigung mit einer erhöhten statistischen Chance für körperliche und psychische Beeinträchtigungen einhergeht [39].

\section{Herausforderung von Umgebungslärm im urbanen Raum}

Umgebungslärm, und hier insbesondere der Verkehrslärm, stellt im urbanen Raum eine große Herausforderung dar $[1,39]$. Durch eine dichte Bauweise in Städten, das hohe Verkehrsaufkommen und die gemischte Nutzung von Wohnen und Gewerbe sind viele Menschen nicht nur durch eine, sondern durch mehrere Schallquellenarten gleichzeitig belastet.

Im Rahmen der repräsentativen Stu-

Abb. $5 \triangleleft$ Wirkungsschema zum geräuschbedingen $\mathrm{Ri}-$ siko von Herz-Kreislauf-Erkrankungen entnommen aus [44]
Straßenverkehrsdauerschallpegels. Mit einem Anstieg von 9\% (KI: 1-14\%) pro $10 \mathrm{~dB} L_{\mathrm{den}}$ des Dauerschallpegels ist das relative Risiko, geräuschbedingt an einer ischämischen Herzkrankheit zu erkranken, für den Luftverkehr vergleichbar hoch [33]. Diese Erkenntnisse stehen auch im Einklang mit neueren Erkenntnissen zum Zusammenhang von Flug- und Straßenverkehrslärm, die nicht mehr in die Metastudie einfließen konnten [34, 35].

\section{Mentale Gesundheit}

Neben den Herz-Kreislauf-Erkrankungen zählen auch psychische Erkrankungen zu den möglichen klinischen Folgen einer dauerhaften Belastung durch Umgebungslärm. Insbesondere die Erforschung des Zusammenhangs zwischen depressiven Störungen und verschiedenen Verkehrslärmquellen ist in den letzten Jahren immer bedeutsamer geworden $[15,36]$. So werden beispielsweise im Rahmen von zwei gro- ßen deutschen Kohortenstudien (HeinzNixdorf-Kohorte, Life Kohorte Leipzig) Analysen und Studien zum Einfluss von Verkehrslärm auf depressive Störungen durchgeführt [37]. Alle Studien weisen auf einen Anstieg depressiver Störungen bei steigenden Schallpegeln hin. In der NORAH-Studie war die unipolare depressive Episode mit einem Anstieg des relativen Risikos von 3,9\% (KI: 2,9-4,9\%) bis $8,9 \%$ (KI: 7,4-10,4\%) pro $10 \mathrm{~dB}$ des jeweiligen Dauerschallpegels sogar das am stärksten repräsentierte Störungsbild [37]. Eine Metaanalyse von Seidler und Kolleginnen [38], die im Rahmen eines laufenden Forschungsvorhabens des Umweltbundesamtes (UBA) $\mathrm{zu}$ den psychischen Auswirkungen von Verkehrslärm durchgeführt wurde, bestätigt die Zusammenhänge. So steigt das relative Risiko, an einer Depression zu erkranken, auf $15 \%$ (KI: 12-18\%) pro $10 \mathrm{~dB} L_{\mathrm{den}}$ Anstieg des Dauerschallpegels für den Fluglärm sowie auf $4 \%$ (KI: 3-6\%) pro $10 \mathrm{~dB} L_{\text {den }}$ Anstieg des Dauerschallpegels für den Straßenver- die zum Umweltbewusstsein in Deutschland untersucht das Umweltbundesamt auch die Mehrfachbelästigung der Menschen durch eine oder mehrere Schallquellenarten im gesamten Bundesgebiet. In Deutschland geben insgesamt $73 \%$ der Menschen an, durch mindestens 2 oder mehrSchallquellenarten belästigt zu sein. Immer noch $51 \%$ der Bevölkerung fühlen sich durch 3 oder mehr Schallquellenarten belästigt. Bei ausschließlicher Betrachtung des Verkehrslärms fühlen sich weiterhin $50 \%$ der Menschen in Deutschland durch 2 oder mehr Verkehrslärmquellenarten belästigt [40].

Die Bedeutsamkeit der Thematik der Mehrfachbelastung durch unterschiedliche Schallquellenarten (auch Gesamtlärmbelastung genannt) spielt insbesondere im urbanen Umfeld eine maßgebende Rolle, daher sieht die Bundesregierung die Einführung einer Gesamtlärmbetrachtung vor [41]. Auch die WHO unterstreicht die Relevanz der Betrachtung von Mehrfachbelastung durch unterschiedliche Schallquellenarten und hält in ihren neuen Leitlinien für Umgebungslärm für die europäische Region fest, dass für eine umfassende Betrachtung der gesundheitlichen Auswirkungen von Umgebungslärm, Mehrfachbelastungen anhand entspre- 
chender Modelle zu berücksichtigen sind [5]. Dies betrifft auch die Mehrfachbelastung durch unterschiedliche Umweltbelastungen, wie beispielsweise Geräusche und Luftschadstoffe oder Vibrationen.

Darüber hinaus stellen auch die Vielzahl und Heterogenität der verschiedenen Schallquellenarten eine Herausforderung dar. $\mathrm{Zu}$ den Hauptbelastungsquellenarten im urbanen Raum zählen neben dem Verkehrslärm auch die Geräusche durch Personen aus der Nachbarschaft, durch Industrie- und Gewerbebetriebe und von Sport- und Freizeitanlagen. Bei diesen Quellenarten ist die Bewertung besonders anspruchsvoll. Dies liegt zum einen daran, dass alle 3 sehr heterogene Geräuschcharakteristika besitzen. Zu den Geräuschen von Personen aus der Nachbarschaft gehören beispielsweise Trittschall, laute Musik, Rufe von Kindern oder nächtliche Unterhaltungen vor dem Fenster. Zu Geräuschen durch Gewerbebetriebe zählen Gespräche von Gästen der benachbarten Gastwirtschaft, aber auch der morgendliche Anlieferverkehr des Supermarkts. Zum anderen gibt es bislang nur wenige Studien, die Rückschlüsse auf Expositions-Wirkungs-Zusammenhänge dieser Schallquellenarten und gesundheitlichen Auswirkungen zulassen.

\section{Maßnahmen zur Minderung der Umgebungslärmbelastung im urbanen Raum}

Die Umgebungslärmrichtlinie ist ein wichtiges Instrument zur Erfassung und Verbesserung der Umgebungslärmsituation in Deutschland (EU-Umgebungslärmrichtlinie 2002/49/EG [3]). Ziel der Richtlinie ist es, den Umgebungslärm $\mathrm{zu}$ vermindern und in bisher ruhigen Gebieten einer Zunahme der Geräuschbelastung vorzubeugen. Dazu wird die Belastung in "Lärmkarten“ erfasst. Auf dieser Grundlage stellen Kommunen unter aktiver Mitwirkung der Öffentlichkeit Lärmaktionspläne auf. In diesen Plänen werden konkrete Maßnahmen zur Lärmminderung geplant. Das mögliche Maßnahmenspektrum für den Lärmschutz im Verkehrsbereich ist vielfältig, die Verantwortung für einzelne
Maßnahmen liegt allerdings bei unterschiedlichen Entscheidungsträgern. Es umfasst sowohl langfristig strategische Ansätze der Verkehrsvermeidung als auch kurzfristig realisierbare Maßnahmen wie den Einsatz lärmmindernder Fahrbahnbeläge.

Eine Auswahl lärmmindernder Maßnahmen wird im Folgenden kurz dargestellt:

Abca. $30 \mathrm{~km} / \mathrm{h}$ ist das Rollgeräusch der Reifen eines Personenkraftfahrzeuges in der Regel lauter als das Motorengeräusch. Daher trägt eine Minderung der Regelgeschwindigkeit auf $30 \mathrm{~km} / \mathrm{h}$ hörbar zur Minderung der Verkehrsgeräusche bei. Im Vergleich zu einer Regelgeschwindigkeit von $50 \mathrm{~km} / \mathrm{h}$ mindert eine Regelgeschwindigkeit von $30 \mathrm{~km} / \mathrm{h}$ in Abhängigkeit vom Lkw-Anteil der entsprechenden Straße den Dauerschallpegel um 2-3 dB. Die Reduzierung der zulässigen Höchstgeschwindigkeit auf Tempo 30 kann nicht nur in Wohngebieten, sondern auch an städtischen Hauptverkehrsstraßen sinnvoll sein. Damit Tempo 30 auf weiteren Straßen (z. B. Bundes-, Landes- und Kreisstraßen) angeordnet werden kann, ist es wichtig, die Straßenverkehrsordnung anzupassen.

Aktuelle Studien verdeutlichen den Zusammenhang zwischen nächtlicher Geräuschbelastung und Herz-KreislaufRisikofaktoren [16, 36]. Dem Schutz der Nachtruhe kommt daher eine hohe Bedeutung zu. Dies gilt vor allem im urbanen Raum aufgrund der großen Zahl an betroffenen Menschen. Maßnahmen zur betrieblichen Beschränkung von Verkehrsträgern können dazu beitragen die nächtliche Geräuschbelastung deutlich zu mindern. Das Umweltbundesamt empfiehlt daher die Einführung des Ruhens des regulären Flugbetriebs an stadtnahen Flughäfen zwischen 22.00 Uhr und 06.00 Uhr.

Die durch Wohnraummangel bedingte Nachverdichtung im urbanen Raum führt dazu, dass die Wohnbebauung wieder dichter an Straßen- und Schienenverkehrswege heranrückt. In kompakten Quartieren mit hoher Bevölkerungsdichte kann baulicher Schallschutz im Neubau beispielsweise dazu beitragen, Verkehrs- und Nachbarschaftslärm zu reduzieren. Die Koexistenz von Wohnbe- bauung und Gewerbe auf engem Raum kann durch die Planung „lärmabgewandter" Fassaden, geschlossener oder Blockbauweisen und durchgesteckter Grundrisse $^{8}$ ermöglicht werden. Kritisch anzumerken bleibt, dass der bauliche Schallschutz durch Maßnahmen des aktiven Schallschutzes im Außenraum Unterstützung finden sollte, da Schallschutzfenster nur die Geräuschsituation im Innenraum verbessern und für die Außenwohnbereiche keinen Schutz bieten. Generell ist daher der Schallschutz an der Quelle allen anderen Maßnahmen zur Lärmminderung vorzuziehen, da er effektiver und meist kosteneffizienter ist.

Neben der Minderung der bestehenden Geräuschbelastungen ist der Erhalt ruhiger Gebiete, gerade in Städten, essenziell. Wohnungsnahe Erholungsgebiete, in denen man „zur Ruhe kommt“, erhöhen die Umwelt- und Lebensqualität von dicht besiedelten und kompakten Städten [42, 43]. Die EU-Umgebungslärmrichtlinie verfolgt neben der Erfassung der Lärmbelastung und deren Minderung auch das Vorsorgeprinzip: Bestehende ruhige Gebiete sollen bewahrt werden. Die konkrete Ausgestaltung der ruhigen Gebiete, z. B. durch Schutzmaßnahmen und Auswahlkriterien, obliegt den für die Lärmaktionsplanung zuständigen Behörden.

\section{Fazit}

Immer mehr Menschen leben in urbanen Räumen in Deutschland. Kompakte Bauweise, Nutzungsmischung, wachsendes Verkehrsaufkommen und eine Vielfalt an unterschiedlichen Geräuschquellen machen Städte besonders laut. So sind mehr als ein Drittel der Menschen, die in Deutschland in einem Ballungsraum leben, ganztägig durchschnittlich einer Verkehrslärmbelastung von mehral $55 \mathrm{~dB} L_{\mathrm{den}}$ ausgesetzt und etwa ein Viertel nachts einer Verkehrslärmbelastung von über $50 \mathrm{~dB} L_{\text {night. }}$ Die WHO empfiehlt -

\footnotetext{
8 Unter "durchgesteckten Grundrissen" wird eine Wohnraumaufteilung verstanden, bei der in jeder Wohnung eine Mindestanzahl der Aufenthaltsräume (z. B. die Hälfte oder mindestens zwei) zu einer geräuschabgewandten Gebäudeseite orientiert sind.
} 
je nach Verkehrslärmquelle - nachts eine durchschnittliche Geräuschbelastung von $40-45 \mathrm{~dB} L_{\text {night }}$ nicht zu überschreiten, um negative Auswirkungen zu vermeiden. Dies bedeutet, dass jede vierte Person, die in Deutschland in einem Ballungsraum lebt, einer Geräuschbelastung ausgesetzt ist, bei der langfristig gesundheitliche Auswirkungen nicht ausgeschlossen werden können.

Grundsätzlich bildet die EU-Umgebungslärmrichtlinie ein rechtliches Rahmenwerk, welches beruhend auf der „Lärmkartierung“ ein breites Spektrum an Maßnahmen bei der Lärmaktionsplanung ermöglicht. Den Kommunen, welche in Deutschland für die Erstellung der Lärmaktionspläne verantwortlich sind, steht jedoch meist nur eine begrenzte Anzahl an technischen Maßnahmen zur Verfügung.

Um die Geräuschbelastung kurz-, mittel- und langfristig im Sinne der empfohlenen Werte der WHO zu senken und einen angemessenen Schutz der Gesundheit der Menschen auch im urbanen Raum zu ermöglichen, bedarf es aus Sicht des Umweltbundesamtes weitreichender Maßnahmen, die durch politische Initiativen zu unterstützen und zu ermöglichen sind. Zu diesen Maßnahmen zählen beispielsweise die Einführung von Tempo 30 als Regelgeschwindigkeit in Städten, das Ruhen des regulären nächtlichen Flugbetriebs an stadtnahen Flughäfen, eine Intensivierung der Bemühung hinsichtlich des baulichen Schallschutzes und der Erhalt ruhiger Gebiete zur lärmarmen Naherholung.

\section{Korrespondenzadresse}

\section{Jördis Wothge}

Fachgebiet I 2.4, Lärmminderung bei Anlagen und Produkten, Lärmwirkungen, Umweltbundesamt

Wörlitzer Platz 1, 06844 Dessau-Roßlau, Deutschland

joerdis.wothge@uba.de

Funding. Open Access funding provided by Projekt DEAL.

\section{Einhaltung ethischer Richtlinien}

Interessenkonflikt. J. Wothge und H. Niemann geben an, dass kein Interessenkonflikt besteht.

Für diesen Beitrag wurden von den Autorinnen keine Studien an Menschen oder Tieren durchgeführt. Für die aufgeführten Studien gelten die jeweils dort angegebenen ethischen Richtlinien.

Open Access. Dieser Artikel wird unter der Creative Commons Namensnennung 4.0 International Lizenz veröffentlicht, welche die Nutzung, Vervielfältigung, Bearbeitung, Verbreitung und Wiedergabe in jeglichem Medium und Format erlaubt, sofern Sie den/die ursprünglichen Autor(en) und die Quelle ordnungsgemäß nennen, einen Link zur Creative Commons Lizenz beifügen und angeben, ob Änderungen vorgenommen wurden.

Die in diesem Artikel enthaltenen Bilder und sonstiges Drittmaterial unterliegen ebenfalls der genannten Creative Commons Lizenz, sofern sich aus der Abbildungslegende nichts anderes ergibt. Sofern das betreffende Material nicht unter der genannten Creative Commons Lizenz steht und die betreffende Handlung nicht nach gesetzlichen Vorschriften erlaubt ist, ist für die oben aufgeführten Weiterverwendungen des Materials die Einwilligung des jeweiligen Rechteinhabers einzuholen.

Weitere Details zur Lizenz entnehmen Sie bitte der Lizenzinformation auf http://creativecommons.org/ licenses/by/4.0/deed.de.

\section{Literatur}

1. Bundes-Immissionsschutzgesetz in der Fassung der Bekanntmachung vom 17. Mai 2013 (BGBI. IS. 1274), das zuletzt durch Artikel 1 des Gesetzes vom 8. April 2019 (BGBI. I S. 432) geändert worden ist, BImSchG (2013).

2. Umweltbundesamt (2020) Indikator: Belastung der Bevölkerung durch Verkehrslärm. Umweltbundesamt:Dessau.https://www.umweltbundesamt. de/indikator-belastung-der-bevoelkerungdurch\#die-wichtigsten-fakten. Zugegriffen: 2. Febr. 2020

3. European Commission (2002) Directive 2002/49/EC of the European Parliament and of the Council of 25 June 2002 relating to the assessment and management of environmental noise. OJ L 189, 18.7.2002:12-25. https://eur-lex. europa.eu/eli/dir/2002/49/oj. Zugegriffen: 2. Febr. 2020

4. WHO (1946by) Preamble to the constitution of the World Health Organization as adopted by the International Health Conference. New York, 19-22 June 1946 World Health Organization, Geneva (signed on 22 July 1946 by the representatives of 61 States and entered into force on 7 April 1948)

5. WHO Regional Office for Europe (2018) Environmental Noise Guidelines for the European Region. WHO Regional Office for Europe, Copenhagen

6. Umweltbundesamt (2019) Umweltbewusstseins studie 2018. Umweltbundesamt, Dessau-Roßlau

7. Guski R, Basner M, Brink M (2012) Gesundheitliche Auswirkungen nächtlichen Fluglärms: aktueller Wissensstand (Literaturauswertung). Literaturstudie. Ruhr-Universität Bochum. Fakultät für Psychologie, Bochum
8. Niemann H, Maschke C, Hecht K (2005) Lärmbedingte Belästigung und Erkrankungsrisiko. Bundesgesundheitsblatt Gesundheitsforschung Gesundheitsschutz 48(3):315-328

9. International Organization for Standardization (2003) Acoustics - Assessment of noise annoyance by means of social and socio-acoustic surveys : Technical Specification ISO/TS15666. International Organization for Standardization, Genf

10. Miedema $\mathrm{H}$, Oudshoorn C (2001) Annoyance from transportation noise: relations with exposure metrics DNL and DENL and their confidence intervals. Environ Mental Health Perspect 109(4):409-441

11. Schreckenberg D, Faulbaum F, Guski $R$ et al (2015) Wirkungen von Verkehrslärm auf die Belästigung und Lebensqualität. In: Gemeinnützige Umwelthaus gGmbH (Hrsg) NORAH (Noise related annoyance cognition and health): Verkehrslärmwirkungen im Flughafenumfeld, Bd. 3. Gemeinnützige Umwelthaus $\mathrm{gGmbH}$, Kelsterbach

12. Guski R, Schreckenberg D, Schuemer R (2017) WHO environmental noise guidelines for the European Region: a systematic review on environmental noise and annoyance. Int J Environ Res Public Health 14(12):1539

13. Héritier $H$, Vienneau D, Foraster et al (2017) Transportation noise exposure and cardiovascular mortality: a nationwide cohort study from Switzerland. Eur JEpidemiol 32(4):307-315

14. Hertenstein E, Feige B, Gmeiner T et al (2019) Insomnia as a predictor of mental disorders: a systematic review and meta-analysis. Sleep Med Rev 43:96-105

15. Röösli M, Wunderli JM, BrinkM, Cajochen C, ProbstHensch N (2019) Die SiRENE-Studie: Verkehrslärm, kardiovaskuläre Sterblichkeit, Diabetes, Schlafstörung und Belästigung. Schweiz Med Forum 19(5):77-82

16. MünzelT, Kroeller-Schön S, Oelze M (2020) Adverse cardiovascular effects of traffic noise with a focus on nighttime noise and the new WHO noise guidelines. Annu Rev Public Health 41:309-328

17. Rechtschaffen A, Kales A (1968) A manual of standardized terminology, techniques and scoring system for sleep stages of human subjects. US National Institute of Neurological Diseases and Blindness, Neurological Information Network, Bethesda

18. Basner M, Samel A, Isermann U (2006) Aircraft noise effects on sleep: application of the results of a large polysomnographic field study. J Acoust Soc Am 52(5):109-123

19. Müller U, Maaß H, Basner M et al (2010) Wirkungsorientierte Bewertung unterschiedlicher Verkehrslärmarten. Verbundprojekt DEUFRAKO/ RAPS - Teilvorhaben DLR: Metaanalyse und Feldstudie - Railway noise (and other modes) Annoyance, Performance, Sleep, Abschlussbericht FKZ: 19U6014B vom 15.09.2010

20. Basner M, McGuire S (2018) WHO environmental noise guidelines for the European region: a systematic review on environmental noise and effects on sleep. Int JEnviron Res Public Health 15(3):519

21. Elmenhorst EM, Griefahn B, Rolny V, Basner M (2019) Comparing the effects of road, railway, and aircraft noise on sleep: exposure — response relationships from pooled data of three laboratory studies. Int JEnviron Res Public Health 16(6):1073

22. Greiser $E$ (2007) Wie verallgemeinerungsfähig sind die Empfehlungen der so genannten FluglärmSynopse und der DLR-Studie zum NachtflugLärm - eine epidemiologische Bewertung. In: Oldiges M (Hrsg) Der Schutz vor nächtlichem 
Fluglärm. Leipziger Schriften zum Umwelt- und Planungsrecht, Bd. 10. Nomos, Baden-Baden

23. Greiser E (2019) Epidemiologische Beurteilung des Gutachtens zur Berücksichtigung eines Maximalpegelkriteriums bei der Beurteilung von Schienenverkehrslärm in der Nacht, sowie der diesem Gutachten zugrunde liegenden Studien und Ermittlung der Erkrankungsrisiken beim Auftreten von drei Aufwachreaktionen in der Nacht. Im Auftrag des Schutzverbandes Umlandgemeinden Flughafen Schönefeld e.V. SOCIUM - Universität Bremen, Abteilung Gesundheit, Pflege und Alterssicherung \& Epi.Consult $\mathrm{GmbH}$, Musweiler

24. EC, European Commission (2004) Position paper on dose-effect relationships for night time noise. Working Group on Health and Socioeconomic Aspects. European Commission, Brussels

25. World Health Organization (WHO) (2009) Night Noise Guidelines (NNGL) for Europe. WHORegional Office for Europe, Copenhagen

26. World Health Organization (WHO) (2011) Burden of diesease from environmental noise; quantification of healthy life years lost in Europe. WHO Regional Office for Europe, Copenhagen

27. Maschke C (2019) Vorhaben VV-0037-2018 Bericht gemäß Teilleistung 1 (AP1 und AP2). Forschungs- und Beratungsbüro Maschke FBB 01 MLUL-101218. Ministerium für Ländliche Entwicklung, Umwelt und Landwirtschaft Brandenburg, Potsdam (122 Seiten)

28. Miedema HME, VosH (2007) Associations between self-reported sleep disturbance and environmental noise based on reanalyses of pooled data from 24 studies. Behav Sleep Med 5(1):1-20

29. Niemann H, Maschke C (2004) WHO LARES Final report Noise effects and morbidity. Berlin: World Health Organisation, t1. http://www.euro.who. int/_data/assets/pdf_file/0015/105144/WHO_ Lares.pdf.Zugegriffen:30. Jan. 2020

30. Klatte M, Bergström K, Spilski J (2014) Wirkungen chronischer Fluglärmbelastung auf kognitive Leistungen und Lebensqualität bei Grundschulkindern. In: Gemeinnützige Umwelthaus gGmbH (Hrsg) NORAH (Noise related annoyance cognition and health): Verkehrslärmwirkungen im Flughafenumfeld, Bd. 1. Gemeinnützige Umwelthaus $\mathrm{gGmbH}$, Kelsterbach

31. Haines M, Stansfeld S, Job R et al (2001) Chronic aircraft noise exposure, stress responses, mental health and cognitive performance in school children. Psychol Med 31(2):265-277

32. Clark C, Paunovic K (2018) WHO environmental noise guidelines for the European Region: a systematic review on environmental noise and cognition. Int J Environ Res Public Health 15(2):E285

33. van Kempen $E$, Casas M, Pershagen G, Foraster M (2018) WHO environmental noise guidelines for the European Region: a systematic review on environmental noise and cardiovascular and metabolic effects: a summary. Int J Environ Res Public Health 15(2):E379

34. Vienneau D, Schindler C, Perez L, Probst-Hensch N, Röösli M (2015) The relationship between transportation noise exposure and ischemic heart disease: a meta-analysis. Environ Res 138:372-380

35. Kaltenbach M, Maschke C, Heß F, Niemann H, Führ M (2016) Health impairments, annoyance and learning disorders caused by aircraft noise: synopsis of the state of current noise research. Int J Environ Prot 6(1):15-46

36. Seidler A, Wagner M, Schubert M et al (2015) Sekundärdatenbasierte Fallkontrollstudie mit vertiefender Befragung. In: Gemeinnützige Um- welthaus gGmbH (Hrsg) NORAH (Noise related annoyance cognition and health): Verkehrslärm

37. Orban E et al (2016) Residential road traffic noise and high depressive symptoms after five years of follow-up: results from the Heinz Nixdorf recall study. Environ Health Perspect 124(5):578-585

38. Seidler A, Schubert M, Romero-Starke Ket al (2019) Traffic noise and mental illness - a systematic review. Environ Epidemiol 3:360

39. Niemann H, Hoebel J, Hammersen F, Laußmann D (2014) Lärmbelästigung -Ergebnisse der GEDAStudie 2012. GBE kompakt 5(4). Robert KochInstitut, Berlin

40. Umweltbundesamt (2020) Gesundheitliche Belastungen durch Umweltverschmutzung und Lärm - Ergebnisse der Umweltbewusstseinsstudien. Umweltbundesamt, Dessau. https:// www.umweltbundesamt.de/sites/default/ files/medien/2378/dokumente/ubs-2018factsheet-gesundheitliche_belastungen_laerm barrierefrei.pdf.Zugegriffen:30. Juni 2020

41. Bundesregierung (Hrsg) (2018) Ein neuer Aufbruch fürEuropa. EineneueDynamikfürDeutschland, Ein neuer Zusammenhalt für unser Land - Koalitionsvertrag zwischen CDU, CSU und SPD, 19. Legislaturperiode. Berlin. https://www.bundesregierung. de/breg-de/themen/koalitionsvertrag-vom-12maerz-2018-975210.Zugegriffen:2. Febr. 2020

42. Gidlöf-Gunnarsson A, Öhrström E (2007) Noise and well-being in urban residential environments: the potential role of perceived availability to nearby green areas. Landsc Urban Plan 83:115-126

43. Shepherd D, Welch D, Dirks KN et al (2013) Do quiet areas afford greater health related quality of life than noisy areas? Int J Environ Res Public Health 10:1284-1303

44. Babisch W, Guski R, Ising H et al (2014) Lärm. In: Wichmann HE, Schlipköter HW, Fülgraff G (Hrsg) Handbuch Umweltmedizin. 52. Erg. Lfg. 6/14. ecomed, Landsberg 